KEK-TH-548,UT-799

November, 1997

\title{
Atmospheric Neutrino Oscillation and Large Lepton-Flavour Violation in the SUSY SU(5) GUT
}

\author{
J. Hisano $^{a)}$, Daisuke Nomura ${ }^{b)}$, and T. Yanagida ${ }^{b)}$ \\ a) Theory Group, KEK, Tsukuba, Ibaraki 305, Japan \\ b) Department of Physics, University of Tokyo, Tokyo 113, Japan
}

\begin{abstract}
The atmospheric neutrino anomaly reported by the super-Kamiokande collaboration suggests existence of a large flavour violating Yukawa coupling in the lepton sector. We discuss lepton-flavour violation at low energies in the framework of the supersymmetric $S U(5)_{G U T}$ model with right-handed neutrinos. We find that for a wide range of parameter space suggested from the atmospheric neutrino anomaly, if the tau-neutrino Yukawa coupling is as large as that of top quark, lower bounds of the branching ratios of $\mu^{+} \rightarrow \mathrm{e}^{+} \gamma$, $\tau^{ \pm} \rightarrow \mu^{ \pm} \gamma$, and the $\mu$-e conversion rate on ${ }_{22}^{48} \mathrm{Ti}$ become around $10^{-14}, 10^{-9}$, and $10^{-16}$, respectively. These reaction rates may be in the region accessible by near future experiments.
\end{abstract}


Small dimensional parameters in physics were often important indications of new, more fundamental physics in the past. A neutrino mass is considered as such an example in the present particle physics. The see-saw mechanism [1] is the most attractive theory for light massive neutrinos in which the smallness of their masses is naturally explained by large Majorana masses of their chiral partners (i.e. righthanded neutrinos).

At present there are two experimental hints of nonvanishing neutrino masses: one is the well-known solar neutrino deficit [2] and the other is the atmospheric neutrino anomaly [3]. A recent report on the atmospheric neutrino from the superKamiokande collaboration [4] has presented convincing evidence that the atmospheric neutrino anomaly is indeed due to neutrino oscillation. From the zenith-angle dependence of the ratio of $\nu_{e}$ and $\nu_{\mu}$ fluxes the super-Kamiokande collaboration [4] has suggested a neutrino mass difference as

$$
\delta m_{\nu_{\mu} \nu_{X}}^{2} \simeq\left(10^{-3}-10^{-2}\right) \mathrm{eV}^{2} .
$$

Provided mass hierarchy $m_{\nu_{\tau}}>m_{\nu_{\mu}}>m_{\nu_{e}}$ it is natural to consider $\nu_{X}=\nu_{\tau}$ while the solar neutrino deficit is explained by the matter oscillation between $\nu_{\mu}$ and $\nu_{e}$ [5]. Thus, the atmospheric neutrino anomaly implies a nonvanishing neutrino mass,

$$
m_{\nu_{\tau}} \simeq(0.03-0.1) \mathrm{eV},
$$

which suggests the presence of right-handed neutrinos at the scale $\sim\left(10^{14}-10^{15}\right)$ $\mathrm{GeV}$. This may support strongly supersymmetric (SUSY) grand unified theories (GUT's), since the mass of the right-handed neutrino is very close to the GUT scale $\simeq 10^{16} \mathrm{GeV}$.

The other important feature of the super-Kamiokande observation is that a large mixing

$$
\sin ^{2} 2 \theta_{\nu_{\mu} \nu_{\tau}} \simeq 1
$$

is required to account for the atmospheric neutrino anomaly. This result already indicates a large lepton-flavour violation (LFV) at the GUT scale. In this letter we point out that Yukawa interactions for the right-handed neutrinos in the 
SUSY $S U(5)_{G U T}$ model induce large LFV at low energies. The present model is an $S U(5)_{G U T^{-}}$-extension of the previous one [6, 7], which yields a significant enhancement in the LFV processes. We find that lower bounds for the branching ratios of $\mu \rightarrow e \gamma$ and $\tau \rightarrow \mu \gamma$, and the $\mu-$ e conversion rate on ${ }_{22}^{48} \mathrm{Ti}$ reach to $10^{-14}$, $10^{-9}$, and $10^{-16}$, respectively, for a reasonable parameter region suggested from the atmospheric neutrino anomaly. This may be accessible by near future experiments.

We should note here that the LFV is also predicted in the minimum SUSY $S U(5)_{G U T}$ model without right-handed neutrinos [8]. However, the reaction rates of the LFV processes are much smaller than predictions in the present model as explained later (see also Ref. [9]).

We introduce singlet chiral multiplets 1 in the SUSY $S U(5)_{G U T}$ model in addition to the ordinary quark and lepton chiral multiplets $\overline{\mathbf{5}}$ and $\mathbf{1 0}$. The singlets correspond to chiral multiplets for the right-handed neutrinos. Yukawa couplings of Higgs multiplets $H(\mathbf{5})$ and $\bar{H}(\overline{\mathbf{5}})$ are given by

$$
W_{S U(5)}=\frac{1}{4} f_{u}^{i j} \mathbf{1 0}_{i} \mathbf{1 0}_{j} H+\sqrt{2} f_{d}^{i j} \mathbf{1 0}_{i} \overline{\mathbf{5}}_{j} \bar{H}+f_{\nu}^{i j} \mathbf{1}_{i} \overline{\mathbf{5}}_{j} H
$$

where $i, j(=1-3)$ are generation indices. Without loss of generality we can always take a basis where $f_{u}^{i j}$ and $f_{\nu}^{i j}$ are diagonal. In this basis the Yukawa couplings can be written as

$$
\begin{aligned}
f_{u}^{i j} & =f_{u_{i}} \mathrm{e}^{i \phi_{u_{i}}} \delta^{i j}, \\
f_{d}^{i j} & =V_{i k}^{\star} f_{d_{k}} U_{k j}^{\dagger}, \\
f_{\nu}^{i j} & =f_{\nu_{i}} \mathrm{e}^{i \phi_{\nu_{i}}} \delta^{i j},
\end{aligned}
$$

where $V$ is the Cabbibo-Kobayashi-Maskawa (CKM) matrix of the quark sector, and $U$ is that of the lepton sector. The additional phases $\phi_{u_{i}}$ and $\phi_{\nu_{i}}$ satisfy $\phi_{u_{1}}+$ $\phi_{u_{2}}+\phi_{u_{3}}=0$ and $\phi_{\nu_{1}}+\phi_{\nu_{2}}+\phi_{\nu_{3}}=0$ In the following discussion these phase parameters are irrelevant to our results. From Eq. (5) we see that LFV interactions

${ }^{1}$ The Yukawa coupling constants $f_{u}, f_{d}$, and $f_{\nu}$ have $2 \times(6+9+9)-3 \times 9=21$ degrees of freedom up to redefinition of fields. These correspond to $(3 \times 3+4 \times 2+2 \times 2)$, in which the first number denotes the number of the eigenvalues of the Yukawa coupling matrices, the second the number of parameters in CKM matrices for quark and lepton sectors, and the third represents the additional phases $\phi_{u_{i}}$ and $\phi_{\nu_{i}}$. 
for left-handed leptons $\left(l_{L}\right)$ in $\overline{\mathbf{5}}$ and right-handed leptons $\left(l_{R}\right)$ in $\mathbf{1 0}$ originate from off-diagonal elements of the matrices $U$ and $V$, respectively.

We assume that Majorana masses for $\mathbf{1}_{i}$ 's are given by the following nonrenormalizable interactions;

$$
W=\frac{c_{i j}}{M_{G}} \mathbf{1}_{i} \mathbf{1}_{j}\langle\Sigma\rangle^{2},
$$

where $\Sigma(\mathbf{2 4})$ is the adjoint Higgs multiplet causing the breaking $S U(5)_{G U T} \rightarrow$ $S U(3) \times S U(2) \times U(1)$ and $M_{G}$ is the gravitational scale $M_{G} \simeq 2.4 \times 10^{18} \mathrm{GeV}$. For simplicity we take $c_{i j}=c \delta_{i j}$ in the present analysis, since our conclusion depends very weakly on the structure of the matrix $c_{i j}$, as far as there is no fine tuning between the matrix $c_{i j}$ and the Yukawa couplings $f_{\nu_{i}}$. In In the case the large neutrino mixing angle $\sin \theta_{\nu_{\mu} \nu_{\tau}} \simeq 1 / \sqrt{2}$ in Eq. (3) corresponds to a large $(3,2)$ element of the matrix $U$. Assuming $c \simeq O(1)$ we restrict our discussion to the case of the Majorana masses for right-handed neutrinos being

$$
M_{N} \simeq\left(10^{13}-10^{15}\right) \mathrm{GeV} .
$$

Notice that the neutrino mass $m_{\nu_{\tau}} \simeq(0.03-0.1) \mathrm{eV}$ requires the considerably large Yukawa coupling constant $f_{\nu_{3}} \simeq(0.1-2.5)$ with $M_{N}$ given by Eq. (7).

Let us introduce soft SUSY-breaking parameters in this model. In terms of the $S U(5)_{G U T}$ multiplets, the soft SUSY-breaking parameters for squarks, sleptons, and Higgses are given by

$$
\begin{aligned}
-\mathcal{L}_{\text {SUSY breaking }}= & \left(m_{\mathbf{1 0}}^{2}\right)_{i}^{j} \widetilde{\mathbf{1 0}} \widetilde{\mathbf{1 0}}_{j}+\left(m_{\overline{\mathbf{5}}}^{2}\right)_{i}^{j} \widetilde{\overline{\mathbf{5}}}^{i \dagger} \widetilde{\overline{\mathbf{5}}}_{j}+\left(m_{\mathbf{1}}^{2}\right)_{i}^{j} \widetilde{\mathbf{i}}^{i \dagger} \widetilde{\mathbf{1}}_{j}+m_{h}^{2} h^{\dagger} h+m_{\bar{h}}^{2} \bar{h}^{\dagger} \bar{h} \\
& +\left\{\frac{1}{4} A_{u}^{i j} \widetilde{\mathbf{1 0}}_{i} \widetilde{\mathbf{1 0}}_{j} h+\sqrt{2} A_{d}^{i j} \widetilde{\mathbf{1 0}} \widetilde{\widetilde{\mathbf{5}}}_{j} \bar{h}+A_{\nu}^{i j} \widetilde{\mathbf{1}}_{i} \widetilde{\mathbf{5}}_{j} h+\text { h.c. }\right\}
\end{aligned}
$$

where $\widetilde{\mathbf{1 0}}_{i}, \widetilde{\overline{\mathbf{5}}}_{i}$, and $\widetilde{\mathbf{1}}_{i}$ are scalar components of the $\mathbf{1 0}_{i}, \overline{\mathbf{5}}_{i}$, and $\mathbf{1}_{i}$ chiral multiplets, respectively, and $h$ and $\bar{h}$ are Higgs bosons. In the minimal supergravity these

\footnotetext{
${ }^{2}$ When there is the following relation between the Yukawa couplings and the Majorana masses of the right-handed neutrinos,

$$
c_{33}: c_{32}: c_{22} \simeq f_{\nu_{3}}^{2}: f_{\nu_{2}} f_{\nu_{3}}: f_{\nu_{2}}^{2},
$$

$\sin ^{2} 2 \theta_{\nu_{\mu} \nu_{\tau}}$ can be close to one even if $(3,2)$ element of $U$ is very small. However, we consider that such a relation between independent couplings and masses is unnatural without some family symmetry 10.

${ }^{3}$ We neglect the Yukawa coupling $\lambda H \Sigma \bar{H}$ and soft SUSY-breaking parameters associated with it, for simplicity. The detailed analysis including such terms will be given in Ref. [11].
} 
parameters are given at the gravitational scale by

$$
\begin{gathered}
\left(m_{\mathbf{1 0}}^{2}\right)_{i}^{j}=\left(m_{\overline{\mathbf{5}}}^{2}\right)_{i}^{j}=\left(m_{\mathbf{1}}^{2}\right)_{i}^{j}=\delta_{j}^{i} m_{0}^{2}, \\
m_{h}^{2}=m_{\bar{h}}^{2}=m_{0}^{2}, \\
A_{u}^{i j}=f_{u}^{i j} a_{0}, A_{d}^{i j}=f_{d}^{i j} a_{0}, A_{\nu}^{i j}=f_{\nu}^{i j} a_{0} .
\end{gathered}
$$

We assume the above relations in the minimal supergravity in the present analysis. If it is not this case, the soft SUSY-breaking parameters at the gravitational scale themselves induce extra contributions to the LFV. Thus, we consider that our results give theoretical lower bounds on the LFV as far as there is no accidental cancellation.

The large Yukawa coupling constants $f_{u_{3}}$ and $f_{\nu_{3}}$ reduce $\left(m_{\mathbf{1 0}}^{2}\right)_{3}^{3}$ and $\left(m_{\overline{\mathbf{5}}}^{2}\right)_{3}^{3}$ significantly through radiative corrections. To make our point clear we neglect the Yukawa coupling constants except for $f_{u_{3}}$ and $f_{\nu_{3}}$ in the following discussion. We include, however, their effects in the numerical calculations. We define magnitudes of the reductions as

$$
\begin{aligned}
R_{\mathbf{1 0}} & \equiv\left(m_{\mathbf{1 0}}^{2}\right)_{1}^{1}-\left(m_{\mathbf{1 0}}^{2}\right)_{3}^{3} \\
R_{\overline{\mathbf{5}}} & \equiv\left(m_{\overline{\mathbf{5}}}^{2}\right)_{1}^{1}-\left(m_{\overline{\mathbf{5}}}^{2}\right)_{3}^{3} .
\end{aligned}
$$

Renormalization group equations (RGE's) for them at the one-loop level are given by

$$
\begin{aligned}
\mu \frac{d R_{\mathbf{1 0}}}{d \mu} & =-\frac{1}{(4 \pi)^{2}} 6 f_{u_{3}}^{2}\left(2\left(m_{\mathbf{1 0}}^{2}\right)_{3}^{3}+\frac{1}{2} m_{h}^{2}\right) \\
\mu \frac{d R_{\overline{\mathbf{5}}}}{d \mu} & =-\frac{1}{(4 \pi)^{2}} 2 f_{\nu_{3}}^{2}\left(\left(m_{\mathbf{1}}^{2}\right)_{3}^{3}+\left(m_{\overline{\mathbf{5}}}^{2}\right)_{3}^{3}+m_{h}^{2}\right)
\end{aligned}
$$

where $\mu$ is the renormalization point. Then, at the GUT scale $\mu \simeq 10^{16} \mathrm{GeV}$ the squark and slepton mass matrices become

$$
\left(m_{\mathbf{1 0}}^{2}\right)=\left(\begin{array}{ccc}
m_{\mathbf{1 0}}^{2} & & \\
& m_{\mathbf{1 0}}^{2} & \\
& & m_{\mathbf{1 0}}^{2}-R_{\mathbf{1 0}}
\end{array}\right), \quad\left(m_{\overline{\mathbf{5}}}^{2}\right)=\left(\begin{array}{ccc}
m_{\overline{\mathbf{5}}}^{2} & & \\
& m_{\overline{\mathbf{5}}}^{2} & \\
& & m_{\overline{\mathbf{5}}}^{2}-R_{\overline{\mathbf{5}}}
\end{array}\right),
$$

where $m_{\mathbf{1 0}}^{2} \equiv\left(m_{\mathbf{1 0}}^{2}\right)_{1}^{1}=\left(m_{\mathbf{1 0}}^{2}\right)_{2}^{2}$ and $m_{\overline{\mathbf{5}}}^{2} \equiv\left(m_{\overline{\mathbf{5}}}^{2}\right)_{1}^{1}=\left(m_{\overline{\mathbf{5}}}^{2}\right)_{2}^{2}$. 
The Yukawa coupling $f_{d}$ in Eq. (5) splits into two parts below the GUT scale: one is $f_{l}$ for leptons and the other $f_{d}$ for down-type quarks. They satisfy a relation $f_{l}=f_{d}$ at the GUT scale. Below the GUT scale we take a basis where the Yukawa coupling matrix $f_{l}$ for leptons is diagonalized as

$$
W_{\mathrm{MSSM}+\nu_{R}}=f_{u_{i}} Q_{i} \bar{U}_{i} H_{2}+V_{i j}^{\star} f_{d_{j}} Q_{i} \bar{D}_{j} H_{1}+f_{l_{i}} \bar{E}_{i} L_{i} H_{1},+f_{\nu_{i}} U^{i j} \bar{N}_{i} L_{j} H_{2}
$$

where $Q, \bar{U}$ and $\bar{D}$ are left-handed quark and right-handed quark chiral multiplets, $L, \bar{E}$, and $\bar{N}$ are those for left-handed leptons, right-handed leptons, and righthanded neutrinos, and $H_{1}$ and $H_{2}$ are those for doublet Higgses. These quarks and leptons are embedded in $\mathbf{1 0}_{i}, \overline{\mathbf{5}}_{i}$, and $\mathbf{1}_{i}$ as

$$
\begin{aligned}
\mathbf{1 0}_{i} & =\left\{Q_{i}, \mathrm{e}^{\left.-i \phi_{u i} \bar{U}_{i}, V^{i j} \bar{E}_{j}\right\}}\right. \\
\overline{\mathbf{5}}_{i} & =\left\{U^{i j} \bar{D}_{j}, U^{i j} L_{j}\right\} \\
\mathbf{1}_{i} & =\left\{\mathrm{e}^{-i \phi_{\nu i}} \bar{N}_{i}\right\}
\end{aligned}
$$

Therefore, the mass matrix for the left-handed sleptons $\left(m_{\tilde{l}_{L}}^{2}\right)$ and that for the righthanded sleptons $\left(m_{\tilde{l}_{R}}^{2}\right)$ are given by

$$
\begin{aligned}
\left(m_{\tilde{l}_{L}}^{2}\right)_{j}^{i} & =U_{j k}^{\dagger}\left(m_{\overline{\mathbf{5}}}^{2}\right)_{l}^{k} U^{l i} \\
& =m_{\overline{\mathbf{5}}}^{2} \delta_{j}^{i}-R_{\overline{\mathbf{5}}} U_{j 3}^{\dagger} U^{3 i}, \\
\left(m_{\tilde{l}_{R}}^{2}\right)_{j}^{i} & =V_{j k}^{\dagger}\left(m_{\mathbf{1 0}}^{2}\right)_{l}^{k} V^{l i} \\
& =m_{\mathbf{1 0}}^{2} \delta_{j}^{i}-R_{\mathbf{1 0}} V_{j 3}^{\dagger} V^{3 i} .
\end{aligned}
$$

As pointed out in Ref. [8] the radiative corrections from the top quark Yukawa couplings produce large LFV masses for right-handed sleptons $\left(\tilde{l}_{R}\right)$. On the other hand, the LFV masses for left-handed sleptons $\left(\tilde{l}_{L}\right)$ induced by the neutrino Yukawa

\footnotetext{
${ }^{4}$ In the SUSY $S U(5)_{G U T}$ model with the right-handed neutrinos the right-handed down-type squarks also have flavour violating masses, and they can contribute to $b \rightarrow s \gamma$ through gluino diagrams. However, the flavour violating masses for the right-handed down-type squarks are the same as those for the left-handed sleptons at the GUT scale, which is constrained from $\operatorname{Br}(\tau \rightarrow$ $\mu \gamma)<4.2 \times 10^{-6}$ [12]. Then, it is difficult to generate a significant contribution to $b \rightarrow s \gamma$ unless we choose an unnatural parameter region.
} 
couplings [13, 6, 7] seem to be relatively smaller than those for $\tilde{l}_{R}$ even if the righthanded tau-neutrino Yukawa coupling is comparable to the top quark Yukawa coupling since they are generated by color-singlet loops.f However, the $(3,2)$ element of $U$ suggested by the atmospheric neutrino anomaly is much larger than that of $V \sim 0.04$, and thus the left-handed sleptons can have larger LFV masses.

In the above discussion, we ignore the bottom quark Yukawa coupling assuming that the vacuum angle $\tan \beta\left(\equiv\left\langle H_{2}\right\rangle /\left\langle H_{1}\right\rangle\right)$ is not large. If it is not the case, the LFV masses for both the left-handed and right-handed sleptons are slightly reduced. However, in this case the LFV event rates become larger as seen later since they are proportional to $\tan ^{2} \beta$ when $\tan \beta \gtrsim 1[6,7,9]$.

Let us now discuss rates of LFV processes. First, we consider $\mu^{+} \rightarrow \mathrm{e}^{+} \gamma$ decay. The amplitude takes a form

$$
T=e \epsilon^{\alpha *}(q) \bar{v}_{\mu}(p) i \sigma_{\alpha \beta} q^{\beta}\left(A_{L} P_{L}+A_{R} P_{R}\right) v_{\mathrm{e}}(p-q),
$$

where $p$ and $q$ are momenta of muon and photon. Then, the decay rate is given by

$$
\Gamma(\mu \rightarrow \mathrm{e} \gamma)=\frac{e^{2}}{16 \pi} m_{\mu}^{3}\left(\left|A_{L}\right|^{2}+\left|A_{R}\right|^{2}\right)
$$

Since the operators in Eq. (17) break not only the $S U(2) \times U(1)$ gauge symmetry but also a chiral symmetry of lepton, $A_{L}$ and $A_{R}$ have to be proportional to a Yukawa coupling constant for lepton and one of two vacuum expectation values of Higgs bosons. Then, the diagrams proportional to $\left\langle H_{2}\right\rangle$ are enhanced by $\tan \beta$ compared with those proportional to $\left\langle H_{1}\right\rangle$, as discussed in Refs. [7, 9]. Furthermore, when both the left-handed and right-handed sleptons have LFV masses, there are diagrams proportional to the tau-lepton mass $m_{\tau}$ (see Fig. 1(a) and (b)). This is a crucial point in the present model. In the SUSY $S U(5)_{G U T}$ model without the right-handed neutrinos, $A_{R}$ is proportional to $m_{\mu}$, and $A_{L}$ is negligible since only the right-handed sleptons have LFV masses [8, 9]. On the other hand, in the SUSY standard model with the right-handed neutrinos, $A_{L}$ is proportional to $m_{\mu}$ and $A_{R}$ negligible [6, []. This is one of the reasons why we obtain relatively large rates for

\footnotetext{
${ }^{5}$ The running effect between the GUT scale and the right-handed neutrino mass makes the LFV masses for left-handed slepton larger. We include this effect in our numerical calculations.
} 
LFV decay processes. A similar enhancement also appears in the SUSY $S O(10)_{G U T}$ model [14]. However, the large mixing angle in the lepton sector required from the atmospheric neutrino anomaly is hard to be explained in the $S O(10)_{G U T}$ model without a fine tuning.

A dominant contribution to $A_{R}$ comes from the diagrams in Fig. 1(a), which are given by

$$
\begin{aligned}
A_{R} \simeq & -\frac{g_{Y}^{2}}{16 \pi^{2}} m_{\tau} M_{\tilde{B}} \mu_{H} \tan \beta\left(m_{\tilde{l}_{L}}^{2}\right)_{3}^{2}\left(m_{\tilde{l}_{R}}^{2}\right)_{1}^{3} \\
& D^{3}\left[\frac{1}{m^{2}} f\left(M_{\tilde{B}}^{2} / m^{2}\right) ; m^{2}\right]\left(m_{\tilde{\mu}_{L}}^{2}, m_{\tilde{\tau}_{L}}^{2}, m_{\tilde{\tau}_{R}}^{2}, m_{\tilde{e}_{R}}^{2}\right) .
\end{aligned}
$$

where $\mu_{H}$ and $M_{\tilde{B}}$ are Higgsino and bino masses, and $m_{\tilde{\mu}_{L}}, m_{\tilde{\tau}_{L}}, m_{\tilde{\tau}_{R}}$, and $m_{\tilde{e}_{R}}$ are left-handed smuon and stau masses and right-handed stau and selectron masses. We have assumed $\tan \beta \gtrsim 1$ and the SUSY breaking scale is larger than $Z$-boson mass $m_{Z}$ in derivation of Eq. (19). Here, a finite-difference function $D[f(x) ; x]$ is defined as

$$
D[f(x) ; x]\left(x_{0}, x_{1}\right) \equiv \frac{1}{x_{0}-x_{1}}\left[f\left(x_{0}\right)-f\left(x_{1}\right)\right],
$$

and then, the $N$-th finite-difference function is given by

$$
D^{N}[f(x) ; x]\left(x_{0}, x_{1}, \cdots, x_{N}\right)=\sum_{i=0}^{N}\left(\prod_{j \neq i} \frac{1}{x_{i}-x_{j}}\right) f\left(x_{i}\right) .
$$

The function $f(x)$ is defined as

$$
f(x)=-\frac{1}{2(1-x)^{3}}\left(1-x^{2}+2 x \log (x)\right) .
$$

We see that the dominant contribution to $A_{R}$ is proportional to $m_{\tau}$. In estimating $A_{R}$ we use the maximal mixing $U^{32} \simeq 1 / \sqrt{2}$ as suggested from the atmospheric neutrino anomaly. D Due to this contribution the decay rate is enhanced by $\left(m_{\tau} / m_{\mu}\right)^{2}\left(U^{32} / V^{32}\right)^{2} \sim 10^{5}$ compared with that in the SUSY SU $(5)_{G U T}$ model without the right-handed neutrinos [8, 9], if the Yukawa coupling for tau neutrino is as large as that of top quark (i.e. $f_{\nu_{3}}=f_{u_{3}}$ ).

\footnotetext{
${ }^{6}$ To calculate $A_{R}$ we also adopt the small angle MSW solution [5] to the solar neutrino problem, which gives $\left|U^{32}\right| \simeq\left|U^{23}\right| \simeq\left|U^{22}\right| \simeq\left|U^{33}\right| \simeq \frac{1}{\sqrt{2}}$ 1.5.
} 
There is a contribution to $A_{L}$ proportional to $m_{\tau}$, which comes from Fig. 1(b), and the explicit form is given by exchanging $L$ and $R$ in Eq. (19). However, since the matrix element $U^{31}$ is unknown, $A_{L}$ has large ambiguity.] Therefore, we take $A_{L}=0$ to evaluate the lower bound of the decay rate of $\mu^{+} \rightarrow \mathrm{e}^{+} \gamma$ from Eq. (18).

Fig. 2 shows the dependence of the lower bound of $\operatorname{Br}(\mu \rightarrow \mathrm{e} \gamma)$ on the righthanded neutrino mass $M_{N}$. In this and the following numerical calculations we adopt the requirement described in Ref. [7] which determines $\mu_{H}$ in terms of other parameters. In this figure $m_{\tilde{\mathrm{e}}_{R}}=150 \mathrm{GeV}, M_{\tilde{B}}=50 \mathrm{GeV}$, and $\mu_{H}>0.1$ Also, we have taken the top quark mass $m_{t}=175 \mathrm{GeV}$ and $m_{\nu_{\tau}}=0.1 \mathrm{eV}$ (solid lines), and $m_{\nu_{\tau}}=0.03 \mathrm{eV}$ (dashed lines). We take $a_{0}=0$ in Eq. (9) for simplicity hereafter. The larger decay rates correspond to the larger $\tan \beta$ as we explained above. To demonstrate this we have taken $\tan \beta=30,10$, and 3 . Note that lower bounds for $\tan \beta=3$ are enhanced by relatively larger $\mu_{H}$ which is determined by the radiative breaking condition for the electroweak symmetry.

For a given tau-neutrino mass, the decay rate is almost proportional to a square of $M_{N}$. It may be surprising that the prediction already exceeds the experimental bound $\left(\operatorname{Br}(\mu \rightarrow \mathrm{e} \gamma)<4.9 \times 10^{-11}[12]\right)$ in a broad parameter region. We have restricted our analysis to the case where the perturbative description of the SUSY GUT is valid up to the gravitational scale. This is the reason why the prediction lines have cuts in the large $M_{N}$ region, $M_{N} \gtrsim 5 \times 10^{14} \mathrm{GeV}\left(2 \times 10^{15} \mathrm{GeV}\right)$ for $m_{\nu_{\tau}}=0.1$ $\mathrm{eV}(0.03 \mathrm{eV})$.

Fig. 3 shows the dependence of the lower bound of $\operatorname{Br}(\mu \rightarrow \mathrm{e} \gamma)$ on $m_{\tilde{\mathrm{e}}_{R}}$ when the tau-neutrino Yukawa coupling is as large as that of the top quark at the gravitational scale (i.e. $f_{\nu_{3}}=f_{u_{3}}$ ). We have fixed $m_{\nu_{\tau}}=0.07 \mathrm{eV}$ and taken $M_{\tilde{B}}=50 \mathrm{GeV}$ (solid lines) and $100 \mathrm{GeV}$ (dashed lines). In this figure the lower bounds for $\tan \beta=3$ are almost degenerate with those for $\tan \beta=10$, and they are slightly larger in larger $m_{\tilde{e}_{R}}$. This comes from the fact that $\mu_{H}$ and $M_{N}$ for $\tan \beta=3$ are larger than those for $\tan \beta=10$. Also, in a region where $m_{\tilde{\mathrm{e}}_{R}}$ is comparable to $M_{\tilde{B}}$, the decay rate is

\footnotetext{
${ }^{7}$ If $\left(g_{2}^{2} / g_{Y}^{2}\right)\left(m_{\mu} / m_{\tau}\right)\left(M_{\tilde{W}} / M_{\tilde{B}}\right)\left(U^{31} / V^{31}\right) \gg 1$ where $M_{\tilde{W}}$ is the wino mass, the wino contribution may be dominant in $A_{L}$.

${ }^{8}$ Our result is insensitive to the sign of $\mu_{H}$ as seen from Eq. (19).
} 
suppressed since the LFV masses are reduced by the radiative corrections from the gaugino loops. On the other hand, if $m_{\tilde{\mathrm{e}}_{R}} \gg M_{\tilde{B}}$, the decay rates are proportional to square of $M_{\tilde{B}}$ as seen in Eq. (19). From this figure, we see that $\operatorname{Br}(\mu \rightarrow \mathrm{e} \gamma)$ is larger than $\sim 10^{-14}$ when the tau-neutrino Yukawa coupling is as large as that for top quark. If the Yukawa coupling for tau neutrino is three times smaller than that of top quark, the lower bound is reduced by a factor $\sim 100$.]

Next, we discuss other LFV processes for muon, $\mu^{+} \rightarrow \mathrm{e}^{+} \mathrm{e}^{-} \mathrm{e}^{+}$and $\mu-\mathrm{e}$ conversion on ${ }_{22}^{48} \mathrm{Ti}$. For these processes the photon penguin contributions in Figs. 1(c) and (d), where large blobs denote the operators in Eq. (17), give dominant amplitudes. Then, we can also derive the lower bounds of $\operatorname{Br}(\mu \rightarrow 3 \mathrm{e})$ and $\mathrm{R}\left(\mu^{-} \rightarrow \mathrm{e}^{-} ;{ }_{22}^{48} \mathrm{Ti}\right){ }^{\mathrm{t}}$ from $\operatorname{Br}(\mu \rightarrow \mathrm{e} \gamma)$, which are given by

$$
\begin{aligned}
\operatorname{Br}(\mu \rightarrow 3 \mathrm{e}) & \simeq 7 \times 10^{-3} \operatorname{Br}(\mu \rightarrow \mathrm{e} \gamma), \\
\mathrm{R}\left(\mu^{-} \rightarrow \mathrm{e}^{-} ;{ }_{22}^{48} \mathrm{Ti}\right) & \simeq 6 \times 10^{-3} \operatorname{Br}(\mu \rightarrow \mathrm{e} \gamma),
\end{aligned}
$$

Thus, we find that $\operatorname{Br}(\mu \rightarrow 3 \mathrm{e})$ and $\mathrm{R}\left(\mu^{-} \rightarrow \mathrm{e}^{-} ;{ }_{22}^{48} \mathrm{Ti}\right)$ are larger than $\sim 10^{-16}$.

Finally, we discuss $\tau^{ \pm} \rightarrow \mu^{ \pm} \gamma$ decay. For this process the amplitude is given by the same form as Eq. (17), and $A_{L}$ and $A_{R}$ depend on $\left(m_{\tilde{l}_{L}}^{2}\right)_{2}^{3}$ and $\left(m_{\tilde{l}_{R}}^{2}\right)_{2}^{3}$, which are both calculable since $U^{32}$ and $V^{32}$ are known. We assume again that the tauneutrino Yukawa coupling is as large as that of the top quark at the gravitational scale $M_{G}$. In this case we find that the amplitude $A_{L}$ is larger than $A_{R}$ in most of the parameter space. This comes from the fact that $\left(m_{\tilde{l}_{L}}^{2}\right)_{3}^{2}$ is larger than $\left(m_{\tilde{l}_{R}}^{2}\right)_{3}^{2}$. When the SUSY breaking scale is larger than $m_{Z}, A_{L}$ is given by

$$
\begin{aligned}
A_{L} \simeq & \frac{g_{2}^{2}}{16 \pi^{2}} m_{\tau} M_{\tilde{W}} \mu_{H} \tan \beta\left(m_{\tilde{L}_{L}}^{2}\right)_{3}^{2} \\
& D\left[D\left[\frac{1}{m^{2}} g\left(M^{2} / m^{2}\right) ; M^{2}\right]\left(M_{\tilde{W}}^{2}, \mu^{2}\right) ; m^{2}\right]\left(m_{\tilde{\mu}_{L}}^{2}, m_{\tilde{\tau}_{L}}^{2}\right),
\end{aligned}
$$

where $M_{\tilde{W}}$ is the wino mass and we have assumed $\tan \beta \gtrsim 1$. The function $g(x)$ is

$$
g(x)=-\frac{1}{4(1-x)^{3}}\left(7-8 x+x^{2}+2(2+x) \log (x)\right) .
$$

\footnotetext{
${ }^{9}$ This smaller value of the Yukawa coupling for tau neutrino also reduces the Majorana mass for the right-handed neutrino by a factor $\sim 10$ for a given $m_{\nu_{\tau}}$.

10 The $\mu$-e conversion rate $\mathrm{R}\left(\mu^{-} \rightarrow \mathrm{e}^{-} ;{ }_{22}^{48} \mathrm{Ti}\right)$ is normalized by the muon capture rate. For the detailed definition of R, see Ref. [12].
} 
Fig. 4 shows the dependence of $\operatorname{Br}(\tau \rightarrow \mu \gamma)$ on $m_{\tilde{\mathrm{e}}_{R}}$. The parameters are taken as in Fig. 3. We see that the branching ratio of $\tau^{ \pm} \rightarrow \mu^{ \pm} \gamma$ is larger than $10^{-9}$ for a broad parameter region.

In conclusion, we have discussed the LFV processes at low energies in the SUSY SU (5) GUT model with the right-handed neutrinos. The atmospheric neutrino anomaly reported by the super-Kamiokande collaboration suggests a large LFV Yukawa coupling at the GUT scale which induces naturally large LFV at low energies through radiative corrections. We have, in fact, found that lower bounds for $\operatorname{Br}(\mu \rightarrow e \gamma), \operatorname{Br}(\tau \rightarrow \mu \gamma)$, and $\mathrm{R}\left(\mu^{-} \rightarrow \mathrm{e}^{-} ;{ }_{22}^{48} \mathrm{Ti}\right)$ reach to $10^{-14}, 10^{-9}$, and $10^{-16}$, respectively, for a wide range of parameter space, if the Yukawa coupling for tau neutrino is as large as that of top quark. This assumption $\left(f_{\nu_{3}}=f_{u_{3}}\right)$ corresponds to $M_{N} \simeq(1-2) \times 10^{14} \mathrm{GeV}$ for $m_{\nu_{\tau}}=0.07 \mathrm{eV}$. This Majorana mass for right-handed neutrino is quite naturally obtained from Eq. (6) with $c \simeq O(1)$.

In the future experiments lower bounds for $\operatorname{Br}(\mu \rightarrow e \gamma)$ and $\mathrm{R}\left(\mu^{-} \rightarrow \mathrm{e}^{-} ;{ }_{22}^{48} \mathrm{Ti}\right)$ are expected to come down to $10^{-14}$ [16, 17, 18] and $10^{-16}$ [19, respectively. We hope that the LFV processes also provide us with strong indication of the presence of right-handed neutrinos.

Acknowledgment

One of the authors (J.H.) would like to thank Y. Okada and Y. Kuno. 


\section{References}

[1] T. Yanagida, in Proceedings of the Workshop on Unified Theory and Baryon Number of the Universe, eds. O. Sawada and A. Sugamoto (KEK, 1979) p.95;

M. Gell-Mann, P. Ramond, and R. Slansky, in Supergravity, eds. P. van Nieuwenhuizen and D. Freedman (North Holland, Amsterdam, 1979).

[2] R. Davis, Jr., D.S. Harmer, and K.C. Hoffman, Phys. Rev. Lett. 20 (1968) 1205;

K.S. Hirata et al., Phys. Rev. Lett. 63 (1989) 16; Phys. Rev. Lett. 65 (1990) 1297.

[3] K.S. Hirata et al., Phys. Lett. B205 (1988) 416; Phys. Lett. B280 (1992) 146;

Y. Fukuda et al., Phys. Lett. B335 (1994) 237;

D. Casper et al., Phys. Rev. Lett. 66 (1991) 2561;

R. Becker-Szendy et al, Phys. Rev. D46 (1992) 3720.

[4] Y. Totsuka, Talk given by Lepton-Photon '97 (Hamburg, July, 1997).

[5] S.P. Mikheyev and A.Y. Smirnov, Yad. Fiz. 42 (1985) 1441 [Sov. J. Nucl. Phys.42(1985)913]; Nuovo Cim. C9 (1986) 17;

L. Wolfenstein, Phys. Rev. D17 (1978) 2369.

[6] J. Hisano, T. Moroi, K. Tobe, M. Yamaguchi, and T. Yanagida, Phys. Lett. B357 (1995) 579.

[7] J. Hisano, T. Moroi, K. Tobe, and M. Yamaguchi, Phys. Rev. D53 (1996) 2442.

[8] R. Barbieri and L.J. Hall, Phys. Lett. B338 (1994) 212.

[9] J. Hisano, T. Moroi, K. Tobe, and M. Yamaguchi, Phys. Lett. B391 (1997) 341; Erratum-ibid B397 (1997) 357.

[10] M. Fukugita, M. Tanimoto, and T. Yanagida, UT-788 (hep-ph/9709388).

[11] J. Hisano and D. Nomura, in preparation.

[12] Particle Data Group, Phys. Rev. D54 (1996) 1.

[13] F. Borzumati and A. Masiero, Phys. Rev. Lett. 57 (1986) 961. 
[14] R. Barbieri, L. Hall, and A. Strumia, Nucl. Phys. B445 (1995) 219.

[15] O. Yasuda, TMUP-HEL-9706 (hep-ph/9706546).

[16] Y. Kuno and Y. Okada, Phys. Rev. Lett. 77 (1996) 434;

Y. Kuno, A. Maki, and Y. Okada, Phys. Rev. D55 (1997) 2517;

Y. Kuno, KEK-Preprint-97-59.

[17] M.D. Cooper, Talk on the fourth KEK typical conference (Tsukuba, Oct, 1996).

[18] S. Orito, private communication.

[19] MECO collaboration, Proposal to Brookhaven National Laboratory AGS (Sep, 1997). 
Figure 1: (a) A contribution to $A_{R}$, proportional to $m_{\tau} \tan \beta$. The large blobs are lepton-flavour violating masses of sleptons. (b) A contribution to $A_{L}$, proportional to $m_{\tau} \tan \beta$. The large blobs are lepton-flavour violating masses of sleptons. (c) A contribution to $\mu^{+} \rightarrow \mathrm{e}^{+} \mathrm{e}^{-} \mathrm{e}^{+}$from lepton-flavour violating dipole terms. The large blob here denotes lepton-flavour violating dipole terms. (d) A contribution to $\mu-\mathrm{e}$ conversion from lepton-flavour violating dipole terms. The large blob denotes lepton-flavour violating dipole terms.

Figure 2: Dependence of the lower bounds of $\operatorname{Br}(\mu \rightarrow \mathrm{e} \gamma)$ on the right-handed neutrino mass $M_{N}$. In this figure the right-handed selectron mass $m_{\tilde{\mathrm{e}}_{R}}=150 \mathrm{GeV}$, the bino mass $M_{\tilde{B}}=50 \mathrm{GeV}$, and the Higgsino mass $\mu_{H}>0$. The solid lines are for $m_{\nu_{\tau}}=0.1 \mathrm{eV}$, and the dashed lines for $m_{\nu_{\tau}}=0.03 \mathrm{eV}$. We take $\tan \beta=30,10$, and 3 , and the larger decay rates correspond to the larger $\tan \beta$.

Figure 3: Dependence of the lower bounds of $\operatorname{Br}(\mu \rightarrow \mathrm{e} \gamma)$ on the right-handed selectron mass $m_{\tilde{\mathrm{e}}_{R}}$ when the tau-neutrino Yukawa coupling is as large as that of the top quark at the gravitational scale $\simeq\left(2 \times 10^{18} \mathrm{GeV}\right)$. We fix $m_{\nu_{\tau}}=0.07 \mathrm{eV}$. Here, the solid lines are for $M_{\tilde{B}}=50 \mathrm{GeV}$ and the dashed lines are for $M_{\tilde{B}}=100 \mathrm{GeV}$. We take $\tan \beta=30,10$, and 3 , and the lines for $\tan \beta=3$ are almost degenerate with those for $\tan \beta=10$, and they are slightly larger in larger $m_{\tilde{\mathrm{e}}_{R}}$.

Figure 4: Dependence of $\operatorname{Br}(\tau \rightarrow \mu \gamma)$ on the right-handed selectron mass $m_{\tilde{e}_{R}}$ when the tau-neutrino Yukawa coupling is as large as that of the top quark at the gravitational scale $\simeq\left(2 \times 10^{18} \mathrm{GeV}\right)$. We fix $m_{\nu_{\tau}}=0.07 \mathrm{eV}$. Here, the solid lines are for $M_{\tilde{B}}=50 \mathrm{GeV}$ and the dashed lines are for $M_{\tilde{B}}=100 \mathrm{GeV}$. We take $\tan \beta=30,10$, and 3 , and the larger decay rates correspond to the larger $\tan \beta$. 


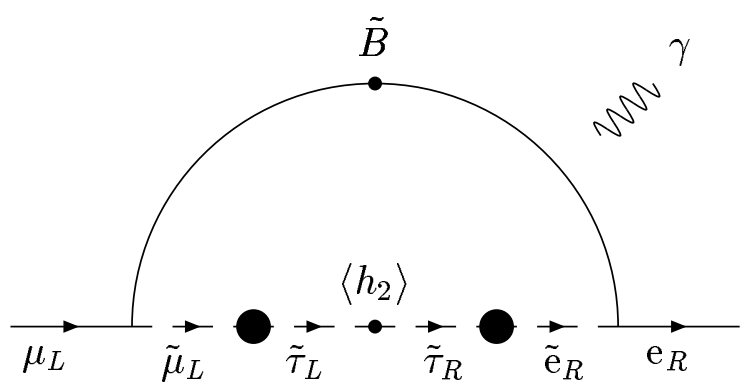

(a)

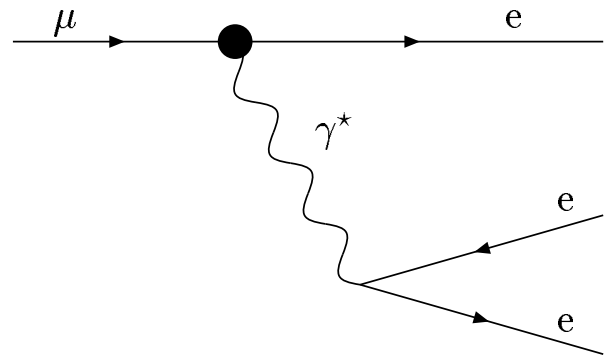

(c)

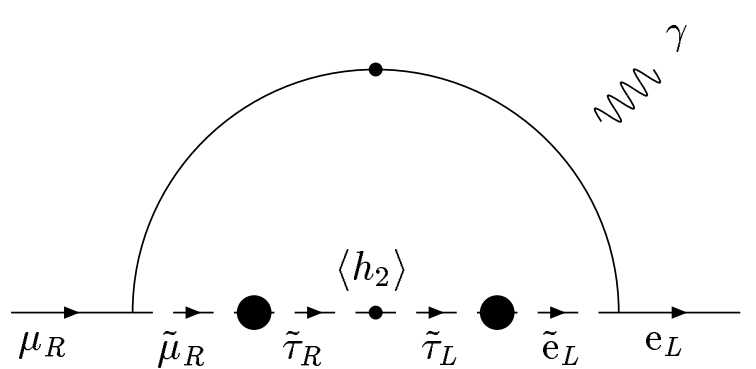

(b)

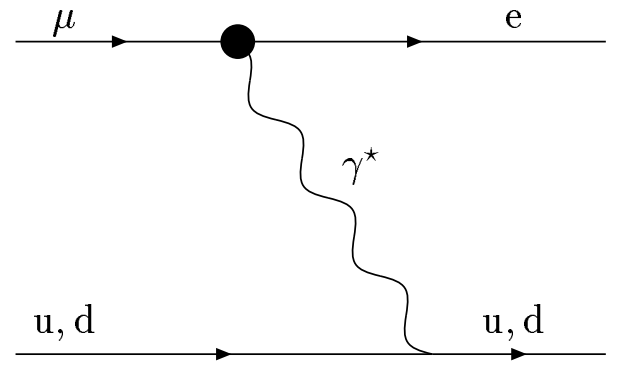

(d)

Fig. 1 


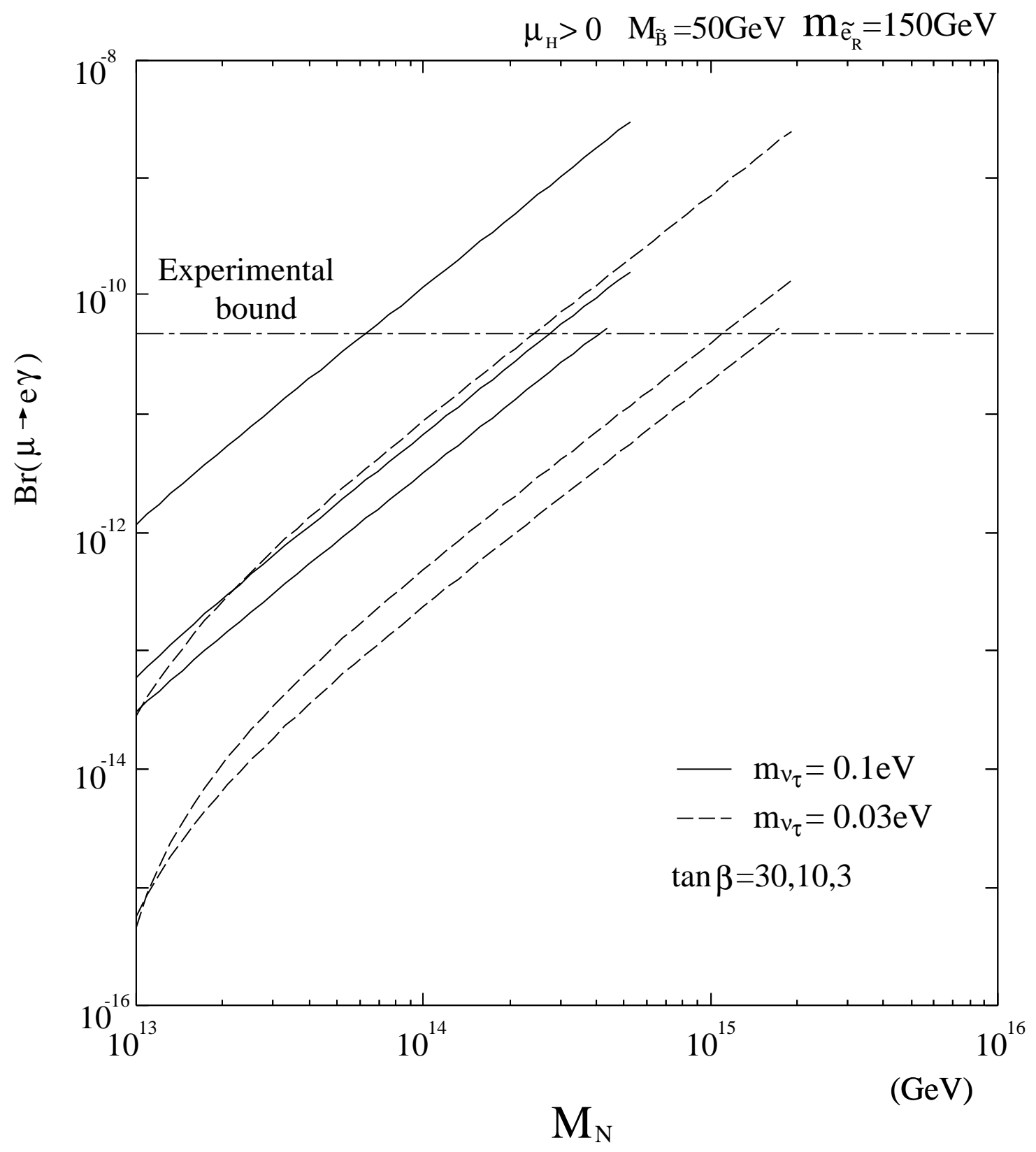

Fig. 2 


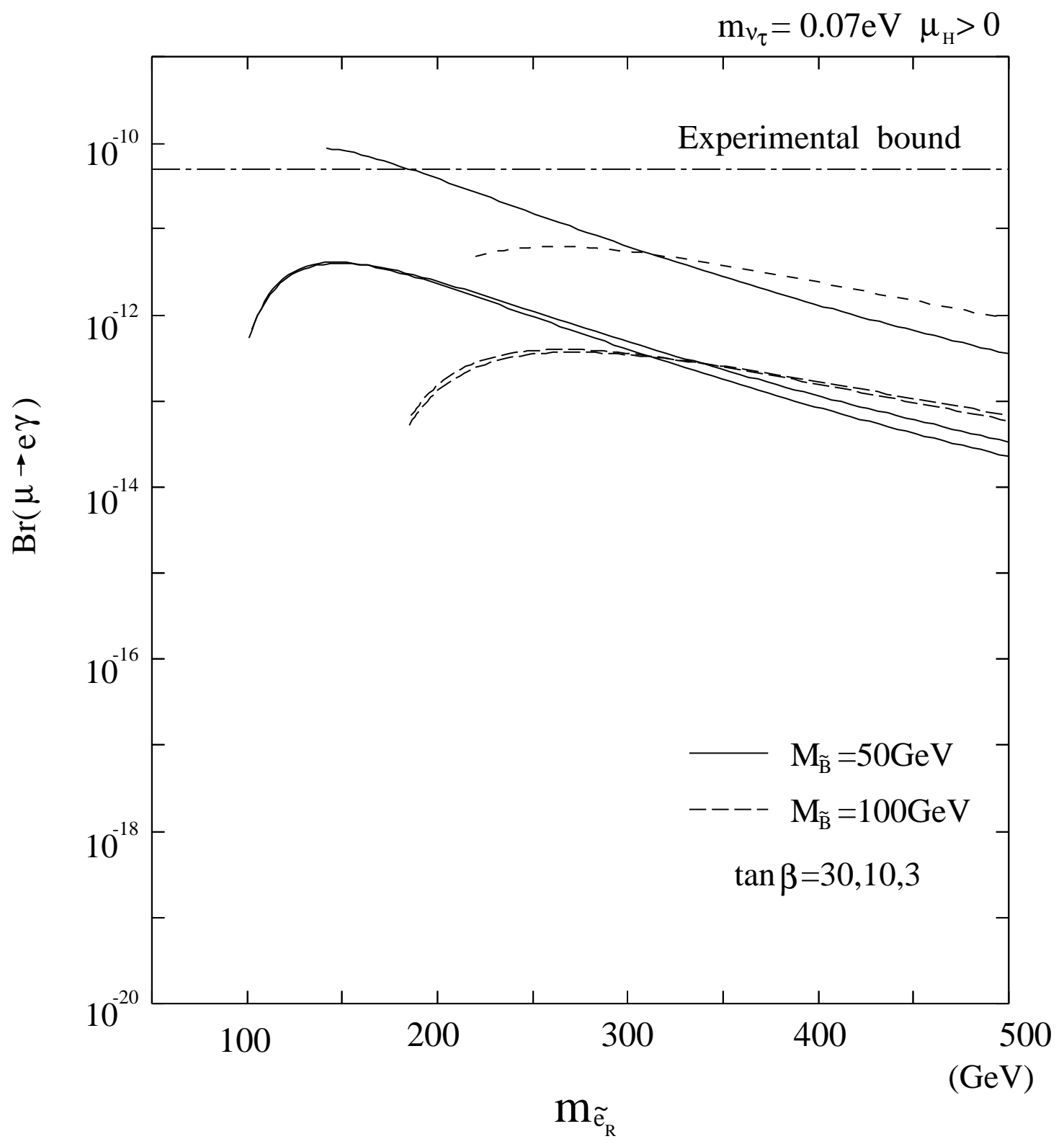

Fig. 3 


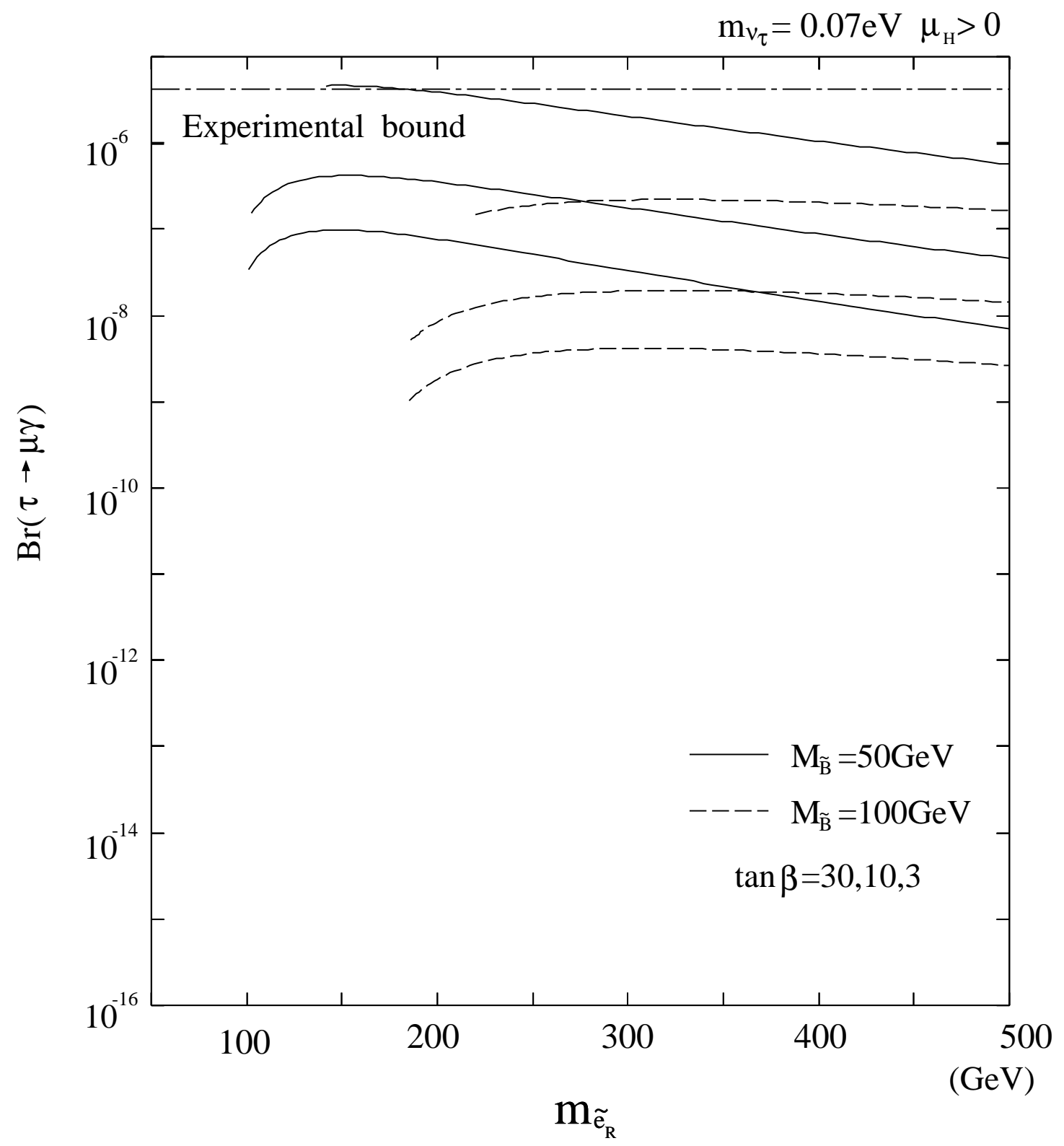

Fig. 4 